\title{
OTIMIZAÇÃO DO PROCESSO FOTO-FENTON UTILIZANDO IRRADIAÇÃO ARTIFICIAL NA DEGRADAÇÃO DO EFLUENTE TÊXTIL SINTÉTICO
}

\author{
Diego Ricieri Manenti ${ }^{1,2}$ \\ Luis Fernando Souza Gomes ${ }^{1}$ \\ Fernando Henrique Borba ${ }^{2}$ \\ Aparecido Nivaldo Módenes ${ }^{2}$ \\ Fernando Rodolfo Espinoza-Quiñones ${ }^{2}$ \\ Soraya Moreno Palácio ${ }^{2}$
}

\begin{abstract}
Resumo: Este trabalho investigou a fotodegradação dos poluentes de um efluente têxtil sintético via processo foto-Fenton. O efluente foi preparado pela mistura de seis corantes. Como fontes de irradiação UV, foram utilizadas três lâmpadas comerciais de mercúrio. As variáveis do processo, $\mathrm{pH}$ inicial e as concentraçôes do íon ferro e peróxido de hidrogênio, foram otimizadas baseado nos resultados de redução da demanda química de oxigênio (DQO) e descoloração obtidos no planejamento fatorial $3^{3}$ completo. Foi obtida uma boa representação dos dados experimentais para um modelo estatístico de quarta ordem, verificado pela aplicação de uma análise de variância. Os dados de degradação do ETS foram ajustados pelo modelo cinético de reação pseudo-primeira ordem. Com apenas 5 minutos de irradiação, o processo foto-Fenton apresentou ótima eficiência na redução da DQO, da turbidez, dos sólidos totais, sólidos totais fixos e sólidos totais voláteis, exceto para os compostos aromáticos, que apresentaram resultados significativos de degradação em 60 minutos.
\end{abstract}

Palavras-chave: corante têxtil; foto-Fenton artificial, planejamento experimental

\begin{abstract}
In this work, the pollutant photo-degradation of a synthetic textile effluent was investigated by using a lab-scale UV-based photo-Fenton reactor. As a synthetic textile effluent a mixture of six dyeing was prepared. As source of UV radiation three mercury commercial lamps were used. A $3^{3}$ full factorial design was applied in order to seek the best values of initial $\mathrm{pH}$, ion iron and hydrogen peroxide concentrations. The photo-Fenton process response was evaluated on the basis of chemical oxygen demand (COD), color and turbidity reduction values. A good representation of experimental data was obtained by a fourth order statistical model, which was checked out by applying a 2-way ANOVA analysis. A pseudo-first order reaction kinetic was applied to fit the substrate biodegradation data. A good performance of UV-based photo-Fenton process was achieved at $5 \mathrm{~min}$, reducing COD, turbidity, total suspended, fixed and volatile solid, except for aromatic compound which was $95 \%$ significantly reduction at $60 \mathrm{~min}$.
\end{abstract}

Keywords: textile dye; photo-Fenton artificial; experimental design

\footnotetext{
Programa de Pós-graduação de Auditoria, Perícia e Gestão Ambiental da Faculdade Assis Gurgacz - FAG

2 Programa de Pós-graduação Stricto Sensu em Engenharia Química da Universidade Estadual do Oeste do Paraná - UNIOESTE. Rua da Faculdade, 645. Jardim La Salle. CEP 85902-000. E-mail: diegomanenti@hotmail.com
} 


\section{INTRODUÇÃO}

O setor têxtil é um dos segmentos industriais mais poluidores das águas superficiais devido aos seus processos consumirem elevadas quantidades de água, que posteriormente são convertidas em efluentes. Estes despejos são caracterizados por altas concentrações de matéria orgânica associados à elevada toxicidade, causada pela presença de corantes, surfactantes, sólidos suspensos e compostos organoclorados (Cisneros, et al., 2002).

A preocupação com a qualidade dos recursos hídricos demanda novas tecnologias para a redução dos poluentes minimizando os impactos ambientais. Nos últimos anos, os processos oxidativos avançados (POAs) são aplicados como alternativa para o tratamento de efluentes de diversas matrizes ambientais (Habibi, et al., 2005). Os POAs são processos em que o principal agente oxidante corresponde ao radical hidroxila $\left({ }^{\circ} \mathrm{OH}\right)$, gerado a partir da combinação entre irradiação UV ou visível com substâncias como o $\mathrm{H}_{2} \mathrm{O}_{2}$ (Lucas \& Peres, 2006), semicondutores como o $\mathrm{TiO}_{2}$ (Habibi, et al., 2005; Garcia, et al., 2009), $\mathrm{ZnO}$ (Rivas, et al., 2008), $\mathrm{Fe}^{2+}$ ou Fe ${ }^{3+}$ (Garcia, et al., 2007; Quici, et al., 2007; Liu, et al., 2007; Durán, et al., 2008), e outras variações e associações. Dentre os POAs destaca-se o processo foto-Fenton (FF), baseado na reação entre sais ferrosos $\left(\mathrm{Fe}^{2+}\right)$ e peróxido de hidrogênio $\left(\mathrm{H}_{2} \mathrm{O}_{2}\right)$, associados à irradiação UV, proporcionando oxidações em tempos bem menores quando comparados aos outros POAs.

O objetivo deste trabalho é investigar a remoção dos poluentes do efluente têxtil pela aplicação do processo foto-Fenton, utilizando luz artificial como fonte de irradiação UV. Os parâmetros de avaliação da eficiência de oxidação foram a demanda química de oxigênio (DQO), a descoloração, a turbidez, os sólidos totais (ST), os sólidos totais fixos (STF), os sólidos totais voláteis (STV), e os compostos aromáticos simples e conjugados.

\section{MATERIAIS E MÉTODOS}

\subsection{MATERIAIS}

O efluente têxtil sintético (ETS) estudado foi preparado pela mistura de seis corantes em água destilada, com concentração de $50 \mathrm{ppm}$. Os corantes Azul FGLE $\left(\mathrm{C}_{40} \mathrm{H}_{23} \mathrm{~N}_{7} \mathrm{Na}_{4} \mathrm{O}_{13} \mathrm{~S}\right)$, Marinho BLE
$\left(\mathrm{C}_{3} \mathrm{H}_{24} \mathrm{~N}_{5} \mathrm{Na}_{3} \mathrm{O}_{13} \mathrm{~S}_{3}\right)$, Escarlate BNLE $\left(\mathrm{C}_{44} \mathrm{H}_{32} \mathrm{~N}_{7}\right.$ $\left.\mathrm{Na}_{4} \mathrm{O}_{16} \mathrm{~S}_{4}\right)$, Preto FR $\left(\mathrm{C}_{44} \mathrm{H}_{32} \mathrm{~N}_{13} \mathrm{Na}_{3} \mathrm{O}_{11} \mathrm{~S}_{3}\right)$, Laranja TGL $\left(\mathrm{C}_{25} \mathrm{H}_{33} \mathrm{ClN}_{6} \mathrm{O}_{6} \mathrm{~S}_{2}\right)$ e Amarelo ARLE $\left(\mathrm{C}_{48} \mathrm{H}_{26} \mathrm{~N}_{8} \mathrm{Na}_{6} \mathrm{O}_{18} \mathrm{~S}_{6}\right)$ foram fornecidos por uma lavanderia industrial localizada na cidade de Toledo - PR, Brasil. $\mathrm{O} \mathrm{H}_{2} \mathrm{O}_{2}(30 \%)$, o $\mathrm{FeSO}_{4} \cdot 7 \mathrm{H}_{2} \mathrm{O}$ (Merck) e outros reagentes são de grade analítica e foram utilizados com prévia purificação.

\subsection{REATOR FOTOQUÍMICO}

O sistema reacional é constituído por três copos becker de $500 \mathrm{~mL}$, três agitadores magnéticos e coletores de alíquotas, montados na forma que a solução fique a aproximadamente $30 \mathrm{~cm}$ de distância das fontes de irradiação (três lâmpadas de alta pressão de mercúrio, Philips - $250 \mathrm{~W}$ ). As lâmpadas são fixadas verticalmente na parte superior de uma caixa de madeira $(80 \mathrm{~cm} \times 80 \mathrm{~cm}$ x $50 \mathrm{~cm})$. Três ventiladores foram posicionados em diferentes posiçôes com o propósito de minimizar os efeitos da temperatura nas lâmpadas. As paredes internas da caixa foram revestidas com papel alumínio evitando perdas de irradiação e mantendo a temperatura interna em torno de $40^{\circ} \mathrm{C}$ durante o processo.

\subsection{PROCEDIMENTO EXPERIMENTAL}

Antes de cada batelada experimental, o $\mathrm{pH}$ inicial do efluente foi ajustado utilizando as soluções padrões de $\mathrm{NaOH}(6 \mathrm{M})$ e $\mathrm{H}_{2} \mathrm{SO}_{4}(3 \mathrm{M})$. É adicionado $500 \mathrm{~mL}$ do efluente no reator seguido das soluções de $\mathrm{H}_{2} \mathrm{O}_{2}$ e $\mathrm{Fe}^{2+}$ nas quantidades determinadas no planejamento experimental. $\mathrm{Na}$ etapa do planejamento experimental o tempo de reação estabelecido foi de 60 minutos, e nas demais foram retiradas alíquotas nos tempos 5, 10, 15, 30, 45, 60, 75 e 90 minutos para análise dos parâmetros físico-químicos.

\subsection{PLANEJAMENTO EXPERIMENTAL}

A influência dos três parâmetros operacionais do reator (POR): $\mathrm{pH}$ inicial e a concentração dos reagentes $\mathrm{H}_{2} \mathrm{O}_{2}$ e $\mathrm{Fe}^{2+}$, foram investigadas baseadas na eficiência de redução da DQO e descoloração, de acordo com um planejamento experimental fatorial $3^{3}$ completo com triplicatas. Os três níveis dos POR foram obtidos em testes preliminares e são apresentados na Tabela 1 . Na análise do processo foto-Fenton, onde os valores dos POR podem influenciar nas respostas experimentais em termos de redução 
dos valores da DQO e descoloração, espera-se que as respostas gráficas 3-D sejam certa função dos níveis dos parâmetros, permitindo a otimização dos valores dos POR. A análise de variância (ANOVA) permite avaliar que tipo de interação entre os POR são estatisticamente significantes para a função resposta.

Tabela 1 - Níveis estudados para os três parâmetros operacionais do reator

\begin{tabular}{lcccc}
\hline \multirow{2}{*}{ POR } & \multirow{2}{*}{ Variáveis } & \multicolumn{3}{c}{ Níveis } \\
\cline { 3 - 5 } & & -1 & 0 & 1 \\
\hline$\left[\mathrm{Fe}^{2+}\right]\left(\mathrm{mg} \mathrm{L}^{-1}\right)$ & $q_{1}$ & 5 & 10 & 15 \\
{$\left[\mathrm{H}_{2} \mathrm{O}_{2}\right]\left(\mathrm{mg} \mathrm{L}^{-1}\right)$} & $q_{2}$ & 50 & 70 & 90 \\
$\mathrm{pH}$ inicial & $q_{3}$ & 2 & 5 & 8 \\
\hline
\end{tabular}

\subsection{DETERMINAÇÕES ANALÍTICAS}

Todas as metodologias utilizadas na determinação dos parâmetros físico-químicos seguiram o Standard Methods (APHA, 2005). O pH foi medido utilizando um $\mathrm{pH}$ metro digital (Tecnal TEC-2). A DQO foi determinada pelo refluxo fechado/método colorimétrico. A turbidez (Nephelometric Turbidity Unit, NTU) foi determinada com um turbidímetro Tecnal, modelo TB1000. A absorvância foi medida utilizando um Espectrofotômetro UV-vis (Shimadzu UV-1610PC) nos comprimentos de onda de máxima absorção $\left(\lambda_{\text {máx }}\right)$ associados aos compostos aromáticos simples $(228,254$ e $284 \mathrm{~nm})$, aos compostos aromáticos conjugados (310 nm), e aos corantes Amarelo $(404 \mathrm{~nm})$, Laranja $(416 \mathrm{~nm})$, Preto $(483 \mathrm{~nm})$, Escarlate (494,5 nm), Azul e Marinho $(588 \mathrm{~nm})$. $\mathrm{Na}$ etapa do planejamento experimental a cor foi mensurada no comprimento de onda $430 \mathrm{~nm}$ (Pt-Co). O percentual de redução da absorvância $(\triangle A b s)$ foi calculado pela Equação (1)

$\Delta A b s(\%)=100 \frac{\left[A b s_{0}^{M}-A b s{ }^{M}\right]}{A b s_{0}^{M}}$

em que: $A b s^{M}{ }_{0}$ e $A b s^{M}$ correspondem aos valores medidos nos comprimentos de onda de máxima absorção de cada composto antes e depois do tratamento, respectivamente. A análise dos teores de sólidos foi realizada através do método gravimétrico e os resultados expressos em $\mathrm{mg} \mathrm{L}^{-1}$. Os Sólidos Totais (ST) foram obtidos pela Equação (2)
$S T\left(m g L^{-1}\right)=\frac{\left(P_{2}-P_{1}\right) 1000}{V_{a}}$

em que: $P_{2}$ corresponde a diferença entre a massa final do recipiente após a evaporação e secagem da amostra, $P_{I}$ é a massa do recipiente vazio e $V_{a}$ corresponde ao volume da amostra $(\mathrm{mL})$. Os Sólidos Totais Fixos (STF) foram calculados pela Equação (3)

$\operatorname{STF}\left(m g L^{-1}\right)=\frac{\left(P_{3}-P_{1}\right) 1000}{V_{a}}$

em que: o sólido obtido na determinação do resíduo total é submetido à ignição de $550 \pm$ $20^{\circ} \mathrm{C}$, e $P_{3}$ corresponde a diferença entre a massa final do recipiente após a calcinação da amostra, o $P_{1}$ é a massa do recipiente vazio e $V_{a}$ é o volume da amostra (mL). E, os Sólidos Totais Voláteis (STV) são obtidos pela diferença entre os sólidos totais e os sólidos totais fixos conforme a Equação (4)

$S T V\left(m g L^{-1}\right)=S T-S T F$

\section{RESULTADOS E DISCUSSŌES}

\subsection{CARACTERIZAÇÃO DO ETS NÃO TRATADO}

As características físico-químicas do ETS não tratado são apresentadas na Tabela 2.

Tabela 2 - Características físico-químicas do ETS não tratado

\begin{tabular}{lc}
\hline Parâmetros & Valores \\
\hline pH & $7,0 \pm 0,2$ \\
Turbidez (NTU) & $47 \pm 3,9$ \\
DQO $\left(\mathrm{mg} \mathrm{L}^{-1}\right)$ & $211 \pm 12$ \\
ST $\left(\mathrm{mg} \mathrm{L}^{-1}\right)$ & $94 \pm 6,2$ \\
STF $\left(\mathrm{mg} \mathrm{L}^{-1}\right)$ & $32 \pm 4,4$ \\
STV $\left(\mathrm{mg} \mathrm{L}^{-1}\right)$ & $62 \pm 5,0$ \\
\hline
\end{tabular}

Os valores da absorvância medidos nos comprimentos de onda de máxima absorção de cada composto presente no ETS são apresentados na Tabela 3. 
Tabela 3 - Valores da absorvância nos comprimentos de onda de máxima absorção dos compostos em estudo presentes no ETS

\begin{tabular}{lcc}
\hline Compostos & $\lambda_{\text {máx }}(\mathrm{nm})$ & Valores \\
\hline \multirow{2}{*}{ Aromáticos Simples } & 228 & 1,180 \\
& 254 & 0,727 \\
Aromáticos conjugados & 284 & 0,675 \\
Amarelo ARLE & 310 & 0,534 \\
Laranja TGL & 404 & 0,775 \\
Cor (PtCo) & 416 & 0,788 \\
Preto FR & 430 & 0,759 \\
Escarlate BNLE & 483 & 0,631 \\
Azul FGLE e Marinho BLE & 594,5 & 0,613 \\
\hline
\end{tabular}

\subsection{ANÁLISE ESTATÍSTICA}

Os resultados obtidos no planejamento experimental fatorial $3^{3}$ completo para o tratamento do ETS são apresentados na Tabela 4. A redução da DQO apresentou variações entre 46,8 e $99,5 \%$, enquanto a descoloração variou entre 80,4 e $99,5 \%$.

Baseado nos resultados da eficiência de redução da DQO e da descoloração combinado as ações das variáveis dos POR, foi proposto um modelo em termos lineares e as interações dos coeficientes, de acordo com a Equação (5), resultando em um bom ajuste entre os valores previstos e observados para a redução da DQO e descoloração, como apresentado na Figura 1.

Tabela 4 - Planejamento fatorial $3^{3}$ completo com triplicatas

\begin{tabular}{cccccccccc}
\hline \multirow{2}{*}{ Exp. } & & & & \multicolumn{3}{c}{ Remoção da DQO (\%) } & \multicolumn{3}{c}{ Descoloração (\%) } \\
\cline { 5 - 10 } & $q_{2}$ & $q_{3}$ & $\mathrm{DQO}_{1}$ & $\mathrm{DQO}_{2}$ & $\mathrm{DQO}_{3}$ & $\mathrm{Des}_{1}$ & Des $_{2}$ & Des $_{3}$ \\
\hline 1 & 5 & 50 & 2 & 77,1 & 78,4 & 76,8 & 82,1 & 83,9 & 82,5 \\
2 & 10 & 50 & 2 & 83,2 & 84,2 & 82,9 & 88,9 & 90,3 & 91 \\
3 & 15 & 50 & 2 & 70,5 & 70,4 & 71 & 90,1 & 87,4 & 88,7 \\
4 & 5 & 70 & 2 & 90,6 & 88,7 & 89,9 & 92,7 & 93 & 94,5 \\
5 & 10 & 70 & 2 & 98,4 & 99,5 & 98,7 & 99,3 & 98,5 & 98,3 \\
6 & 15 & 70 & 2 & 85,8 & 83,9 & 84,8 & 98,2 & 99,1 & 99,4 \\
7 & 5 & 90 & 2 & 59,9 & 59,2 & 61,2 & 93,8 & 92 & 94 \\
8 & 10 & 90 & 2 & 63,1 & 62,6 & 62,1 & 99,4 & 99,5 & 99 \\
9 & 15 & 90 & 2 & 55,2 & 54,2 & 55,6 & 95 & 93,2 & 95,1 \\
10 & 5 & 50 & 5 & 75 & 75,4 & 76,3 & 82,9 & 83,6 & 82,2 \\
11 & 10 & 50 & 5 & 86,6 & 87,2 & 86,1 & 86,6 & 85,9 & 87,2 \\
12 & 15 & 50 & 5 & 72,3 & 73,5 & 72,8 & 88,1 & 87,4 & 86,9 \\
13 & 5 & 70 & 5 & 83,7 & 82,1 & 83,5 & 93,3 & 93,5 & 94,2 \\
14 & 10 & 70 & 5 & 95,3 & 96,5 & 95,7 & 97,2 & 97,9 & 98,9 \\
15 & 15 & 70 & 5 & 81,1 & 79,8 & 81,9 & 99 & 99,2 & 99,4 \\
16 & 5 & 90 & 5 & 57,5 & 57,4 & 58,4 & 96,2 & 94,1 & 95 \\
17 & 10 & 90 & 5 & 60,2 & 59,1 & 58,7 & 96,7 & 95,7 & 97,2 \\
18 & 15 & 90 & 5 & 56 & 56,5 & 57,1 & 97,6 & 98,2 & 99 \\
19 & 5 & 50 & 8 & 66,8 & 66,7 & 67,2 & 82 & 80,4 & 81,9 \\
20 & 10 & 50 & 8 & 69,4 & 68,3 & 69 & 83 & 83,2 & 84,3 \\
21 & 15 & 50 & 8 & 65,6 & 64,9 & 65,2 & 87 & 85,6 & 87,1 \\
22 & 5 & 70 & 8 & 80,2 & 80,9 & 79,4 & 91,1 & 89,9 & 90,3 \\
23 & 10 & 70 & 8 & 89,9 & 89,2 & 90 & 89,5 & 90,4 & 87 \\
24 & 15 & 70 & 8 & 78,2 & 77,9 & 78,5 & 90 & 93 & 92,1 \\
25 & 5 & 90 & 8 & 53,4 & 55 & 54,5 & 89 & 90,8 & 88,7 \\
26 & 10 & 90 & 8 & 59,2 & 59,9 & 59 & 95 & 92,5 & 93,9 \\
27 & 15 & 90 & 8 & 47,5 & 46,8 & 47,1 & 96,3 & 94,7 & 97 \\
\hline
\end{tabular}




$$
\begin{aligned}
& R=a_{0}+\sum_{i=1}^{N} a_{i} a_{j}+\sum_{i=1}^{N} \sum_{j=1}^{N} b_{j} q_{i} q_{j}+ \\
& \sum_{i=1}^{N} \sum_{j=1}^{N} \sum_{k=1}^{N} w_{i j k} q_{i} q_{j} q_{k}+\sum_{i=1}^{N} \sum_{j=1}^{N} v_{j} q_{i}^{2} q_{j}^{2}
\end{aligned}
$$

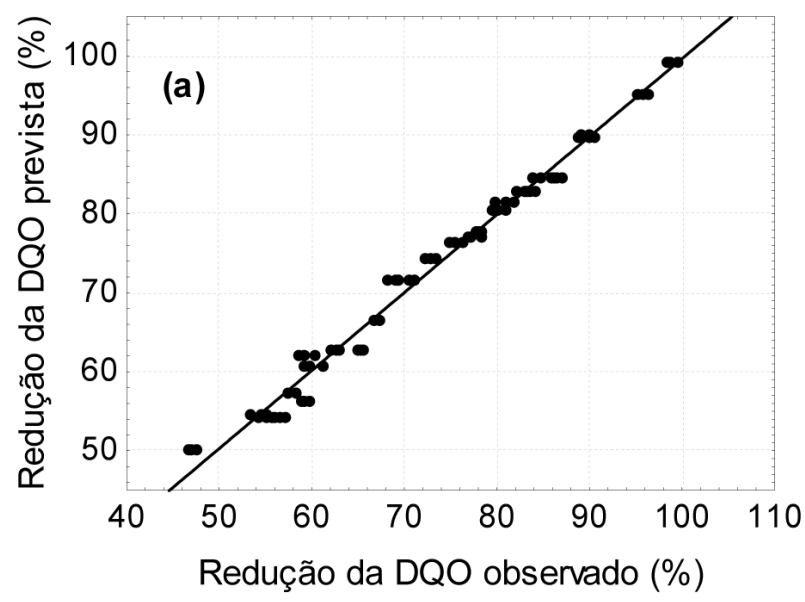

em que: $R$ é a resposta experimental; $q$ é o valor do POR ajustado; $a_{0}$ é a constante; $a$ é o coeficiente dos termos lineares ajustados; $b, w$ e $v$ são os coeficientes associados com as interaçóes lineares e quadráticas entre os valores dos POR ajustados; e $N$ é o número do POR.

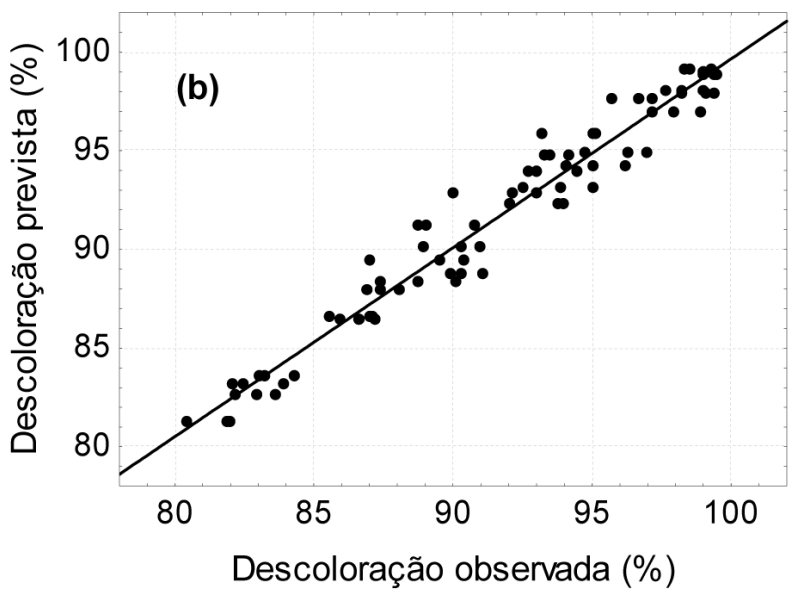

Figura 1 - Correlação entre os valores observados de redução da DQO (a) e descoloração (b) e seus valores correspondentes previstos pelo modelo estatístico proposto

As respostas experimentais, baseadas nos valores da DQO e descoloração, permitiram o desenvolvimento de um modelo estatístico, com o ajuste linear e quadrático dos coeficientes associados com os valores dos POR, conforme apresen- tados nas Tabelas 5 e 6 . A significância dos efeitos dos POR e suas possíveis ações combinadas são verificadas aplicando a análise de variâncias (2-way ANOVA), utilizando o software Statistica ${ }^{\circledR}$, como apresentado nas Tabelas 7 e 8.

Tabela 5 - Valores do ajuste linear e da interação dos coeficientes do modelo previsto para a redução da DQO, com nível de significância de $95 \%(\mathrm{p}<5 \%)$ e $\mathrm{r}^{2}=0,98762$

\begin{tabular}{lccccc}
\hline Ações dos parâmetros & Coeficientes & Valor & Desvio padrão & $t_{\text {exp }}$ & $p(\%)$ \\
\hline $\mathrm{q}_{0}$ & $a_{0}$ & 72,633 & 0,194 & 375,065 & $<0,01$ \\
$\mathrm{q}_{1}$ & $a_{1}$ & $-3,744$ & 0,474 & $-7,894$ & $<0,01$ \\
$\left(\mathrm{q}_{1}\right)^{2}$ & $b_{11}$ & 8,494 & 0,411 & 20,677 & $<0,01$ \\
$\mathrm{q}_{2}$ & $a_{2}$ & $-17,274$ & 0,474 & $-36,416$ & $<0,01$ \\
$\left(\mathrm{q}_{2}\right)^{2}$ & $b_{22}$ & 21,278 & 0,411 & 51,795 & $<0,01$ \\
$\mathrm{q}_{3}$ & $a_{3}$ & $-8,081$ & 0,474 & $-17,037$ & $<0,01$ \\
$\left(\mathrm{q}_{3}\right)^{2}$ & $b_{33}$ & 2,478 & 0,411 & 6,032 & $<0,01$ \\
$\mathrm{q}_{1} \times \mathrm{q}_{2}$ & $b_{12}$ & $-0,389$ & 0,581 & $-0,669$ & 50,57 \\
$\mathrm{q}_{1} \times\left(\mathrm{q}_{2}\right)^{2}$ & $w_{122}$ & 0,550 & 0,503 & 1,093 & 27,85 \\
$\left(\mathrm{q}_{1}\right)^{2} \times \mathrm{q}_{2}$ & $w_{112}$ & $-1,461$ & 0,503 & $-2,904$ & 0,51 \\
$\left(\mathrm{q}_{1}\right)^{2} \times\left(\mathrm{q}_{2}\right)^{2}$ & $v_{12}$ & 2,608 & 0,436 & 5,986 & $<0,01$ \\
$\mathrm{q}_{1} \times \mathrm{q}_{3}$ & $b_{13}$ & 1,000 & 0,581 & 1,721 & 9,02 \\
$\mathrm{q}_{1} \times\left(\mathrm{q}_{3}\right)^{2}$ & $w_{133}$ & 1,283 & 0,503 & 2,551 & 1,32 \\
$\left(\mathrm{q}_{1}\right)^{2} \times \mathrm{q}_{3}$ & $w_{113}$ & $-0,672$ & 0,503 & $-1,336$ & 18,64 \\
$\left(\mathrm{q}_{1}\right)^{2} \times\left(\mathrm{q}_{3}\right)^{2}$ & $v_{13}$ & 0,733 & 0,436 & 1,683 & 9,74 \\
$\mathrm{q}_{2} \times \mathrm{q}_{3}$ & $b_{23}$ & 2,261 & 0,581 & 3,892 & $<0,01$
\end{tabular}




\begin{tabular}{lccccc}
$\mathrm{q}_{2} \times\left(\mathrm{q}_{3}\right)^{2}$ & $w_{233}$ & $-2,403$ & 0,503 & $-4,776$ & $<0,01$ \\
$\left(\mathrm{q}_{2}\right)^{2} \times \mathrm{q}_{3}$ & $w_{223}$ & $-0,281$ & 0,503 & $-0,558$ & 57,9 \\
$\left(\mathrm{q}_{2}\right)^{2} \times\left(\mathrm{q}_{3}\right)^{2}$ & $v_{23}$ & $-2,079$ & 0,436 & $-4,772$ & $<0,01$ \\
\hline
\end{tabular}

Tabela 6 - Valores do ajuste linear e da interação dos coeficientes do modelo previsto para a descoloração, com nível de significância de $95 \%(\mathrm{p}<5 \%)$ e $\mathrm{r}^{2}=0,95684$.

\begin{tabular}{lccccc}
\hline Ações dos parâmetros & Coeficientes & Valor & Desvio padrão & $t_{\text {exp }}$ & $p(\%)$ \\
\hline $\mathrm{q}_{0}$ & $a_{0}$ & 91,823 & 0,145 & 634,307 & $<0,01$ \\
$\mathrm{q}_{1}$ & $a_{1}$ & 4,304 & 0,355 & 12,137 & $<0,01$ \\
$\left(\mathrm{q}_{1}\right)^{2}$ & $b_{11}$ & 1,504 & 0,307 & 4,897 & $<0,01$ \\
$\mathrm{q}_{2}$ & $a_{2}$ & 9,570 & 0,355 & 26,990 & $<0,01$ \\
$\left(\mathrm{q}_{2}\right)^{2}$ & $b_{22}$ & 4,426 & 0,307 & 14,413 & $<0,01$ \\
$\mathrm{q}_{3}$ & $a_{3}$ & $-4,193$ & 0,355 & $-11,824$ & $<0,01$ \\
$\left(\mathrm{q}_{3}\right)^{2}$ & $b_{33}$ & 1,881 & 0,307 & 6,127 & $<0,01$ \\
$\mathrm{q}_{1} \times \mathrm{q}_{2}$ & $b_{12}$ & $-0,794$ & 0,434 & $-1,830$ & 7,22 \\
$\mathrm{q}_{1} \times\left(\mathrm{q}_{2}\right)^{2}$ & $w_{122}$ & $-0,153$ & 0,376 & $-0,406$ & 68,6 \\
$\left(\mathrm{q}_{1}\right)^{2} \times \mathrm{q}_{2}$ & $w_{112}$ & 0,197 & 0,376 & 0,524 & 60,2 \\
$\left(\mathrm{q}_{1}\right)^{2} \times\left(\mathrm{q}_{2}\right)^{2}$ & $v_{12}$ & $-0,624$ & 0,326 & $-1,915$ & 60,16 \\
$\mathrm{q}_{1} \times \mathrm{q}_{3}$ & $b_{13}$ & 0,056 & 0,434 & 0,128 & 89,87 \\
$\mathrm{q}_{1} \times\left(\mathrm{q}_{3}\right)^{2}$ & $w_{133}$ & 0,089 & 0,376 & 0,236 & 81,39 \\
$\left(\mathrm{q}_{1}\right)^{2} \times \mathrm{q}_{3}$ & $w_{113}$ & $-2,306$ & 0,376 & $-6,130$ & $<0,01$ \\
$\left(\mathrm{q}_{1}\right)^{2} \times\left(\mathrm{q}_{3}\right)^{2}$ & $v_{13}$ & $-0,428$ & 0,326 & $-1,313$ & 19,4 \\
$\mathrm{q}_{2} \times \mathrm{q}_{3}$ & $b_{23}$ & 0,406 & 0,434 & 0,934 & 35,40 \\
$\mathrm{q}_{2} \times\left(\mathrm{q}_{3}\right)^{2}$ & $w_{233}$ & 1,064 & 0,376 & 2,829 & $<0,01$ \\
$\left(\mathrm{q}_{2}\right)^{2} \times \mathrm{q}_{3}$ & $w_{223}$ & $-1,831$ & 0,376 & $-4,867$ & $<0,01$ \\
$\left(\mathrm{q}_{2}\right)^{2} \times\left(\mathrm{q}_{3}\right)^{2}$ & $v_{23}$ & 1,043 & 0,326 & 3,202 & $<0,01$ \\
\hline
\end{tabular}

Baseado no total de 27 experimentos foi construído os gráficos 3-D em função das respostas $(\mathrm{R})$, e os dados experimentais foram previstos utilizando um modelo de quarta ordem (ver Eq. (5)) para os valores de redução da DQO e descoloração, apresentando suas dependências em relação aos valores dos POR.

A análise da ANOVA mostrou que o modelo previsto (Equação (5)) é válido, resultando em uma reprodução muito boa das respostas experimentais conforme apresentado nas Tabelas 7 e 8.

A análise da ANOVA apresentou nível de confiança de 99,99\%, quando o nível de significância foi calculado na proporção do erro da média quadrática devido a regressão e os resíduos, sendo $<0,01$. A análise estatística do planejamento fatorial $3^{3}$ completo apresentou os valores dos POR que tem influência significativa na redução da DQO e descoloração, proporcionando os valo- res estimados dos coeficientes $a_{1}, a_{2}$ e $a_{3}$. Todos os parâmetros em termos lineares e quadráticos têm influência significativa $(\mathrm{p}<0,01 \%)$ para a eficiência do processo na redução da DQO e descoloração. $\mathrm{O}$ efeito negativo $\left(a_{3}<0\right.$, ver Tabelas 5 e 6) em relação ao nível do $\mathrm{pH}$ inicial, sugere que a melhor eficiência do processo FF para a redução dos poluentes é obtida com $\mathrm{pH}$ inicial em meio ácido, resultados similares foram encontrados em outros trabalhos que afirmam a melhor eficiência dos POAs nas mesmas condições (Kajitvichyanukul \& Jamroensan, 2008; Knittel \& Schollmeyer, 2008; Makhotkina, et al., 2008; Primo, et al., 2008). A ação do $\mathrm{Fe}^{2+}$ exerce influência significativa na eficiência do processo FF para a redução da DQO, apresentando efeito negativo para os termos lineares $\left(a_{1}=-3,744\right)$ e efeito positivo para os termos quadráticos $\left(b_{11}=8,494\right)$ como apresentado na Tabela 5. 
Tabela 7 - Teste da análise de variância (2-way ANOVA) do modelo previsto para a redução da DQO

\begin{tabular}{lcccccc}
\hline & \multirow{2}{*}{$\begin{array}{c}\text { Soma dos } \\
\text { quadrados }\end{array}$} & $\begin{array}{c}\text { Graus de } \\
\text { liberdade }\end{array}$ & Média dos & \multicolumn{2}{c}{$\mathrm{F}$} & \multirow{2}{*}{$\begin{array}{c}\text { Nível de } \\
\text { quadrados }\end{array}$} \\
\cline { 5 - 6 } & & Calculado & Estatístico & significância (\%) \\
\hline Regressão & 15025,64 & 18 & 834,75 & 274,588 & 1,8 & $<0.01$ \\
Resíduos & 188,34 & 62 & 3,04 & & & \\
Total & 15213,98 & 80 & & & & \\
\hline
\end{tabular}

Tabela 8 - Teste da análise de variância (2-way ANOVA) do modelo previsto para a descoloração

\begin{tabular}{|c|c|c|c|c|c|c|}
\hline & \multirow{2}{*}{$\begin{array}{l}\text { Soma dos } \\
\text { quadrados }\end{array}$} & \multirow{2}{*}{$\begin{array}{l}\text { Graus de } \\
\text { liberdade }\end{array}$} & \multirow{2}{*}{$\begin{array}{l}\text { Média dos } \\
\text { quadrados }\end{array}$} & \multicolumn{2}{|c|}{$\mathrm{F}$} & \multirow{2}{*}{$\begin{array}{c}\text { Nível de } \\
\text { significância } \\
(\%)\end{array}$} \\
\hline & & & & Calculado & Estatístico & \\
\hline Regressão & 2333,02 & 18 & 129,61 & 76,36 & 1.8 & $<0.01$ \\
\hline Resíduos & 105,24 & 62 & 1,697 & & & \\
\hline Total & 2438,27 & 80 & & & & \\
\hline
\end{tabular}

Resultados similares ocorrem para a ação do $\mathrm{H}_{2} \mathrm{O}_{2}$, porém, em maiores proporções, sendo o efeito negativo para os termos lineares $\left(a_{2}=\right.$ $-17,274)$ e o efeito positivo para os termos quadráticos $\left(b_{22}=21,278\right)$ na redução da DQO (ver Tabela 5). Para a descoloração, os efeitos dos termos lineares e quadráticos do $\mathrm{Fe}^{2+}$ e do $\mathrm{H}_{2} \mathrm{O}_{2}$ são positivos (ver Tabela 6).

Combinando $\mathrm{Fe}^{2+}$ e $\mathrm{H}_{2} \mathrm{O}_{2}$, estes apresentam significância na eficiência do processo apenas para a ação entre os termos quadráticos $\left(v_{12}\right.$ $=2,608, \mathrm{p}<0,01 \%)$, e a combinação destes com o $\mathrm{pH}$ inicial apresenta influência significativa (p $<0,01 \%)$ apenas para as ações com o $\mathrm{H}_{2} \mathrm{O}_{2}\left(b_{23}\right.$, $w_{233}$ e $v_{23}$ ) como apresentado na Tabela 5. Resultados similares são verificados para a descoloração, quando apenas as interações quadráticas entre o $\mathrm{pH}$ inicial e o $\mathrm{H}_{2} \mathrm{O}_{2}\left(w_{233}, w_{223}\right.$ e $v_{23}$, ver Tabela 6) apresentam influência significativa (p $<0,01 \%)$.

Os resultados apresentados podem ser visualizados na Figura 2. As melhores condiçōes do processo FF são com o $\mathrm{pH}$ inicial 2,3 e as concentraçôes de $\mathrm{Fe}^{2+}=9,3 \mathrm{mg} \mathrm{L}^{-1} \mathrm{e} \mathrm{H}_{2} \mathrm{O}_{2}=65,5 \mathrm{mg} \mathrm{L}^{-1}$, obtendo cerca de $97 \%$ de eficiência na redução da DQO.

\subsection{EFEITO DO TEMPO DE REAÇÃO NA DEGRADAÇÃO DO ETS}

A eficiência de oxidação foi monitorada com a redução da DQO, da turbidez, dos ST, STF, STV e em nove comprimentos de onda $(228,254$, 284, 310, 404, 416, 483, 494,5 e $588 \mathrm{~nm}$ ). Estes comprimentos de onda foram selecionados por estarem relacionados aos comprimentos de onda de máxima absorção dos compostos aromáticos simples $(228 \mathrm{~nm})$, compostos aromáticos conjugados (254, 284 e $310 \mathrm{~nm})$ e aos corantes Amarelo (404 $\mathrm{nm})$, Laranja (416 nm), Preto (483 nm), Escarlate (494,5), Azul e Marinho (588 nm).

Utilizando as melhores condições operacionais do processo FF encontradas no planejamento fatorial $3^{3}$ completo, foi obtido o espectro UV/vis em função do ETS não-tratado e tratado com o tempo de degradação estabelecido em 60 minutos conforme apresentado na Figura 3. O espectro apresenta que com 60 minutos de irradiação não há mais absorvâncias nos comprimentos de onda referentes aos corantes e que os compostos aromáticos são mais persistentes. A redução da absorvância indica a fragmentação das estruturas orgânicas com completa descolo- 
ração do ETS e a perda parcial dos compostos aromáticos. Estes resultados podem ser explicados pela seguinte reação

$$
\begin{aligned}
& \mathrm{Fe}^{2+} / \mathrm{H}_{2} \mathrm{O}_{2} / \mathrm{UV} \\
& \mathrm{Fe}(\mathrm{OH})^{2+} \rightarrow \mathrm{Fe}^{3+}+\mathrm{OH}^{\cdot} \\
& \mathrm{Fe}^{2+}+\mathrm{H}_{2} \mathrm{O}_{2} \rightarrow \mathrm{Fe}^{3+}+\mathrm{OH}^{-}+\mathrm{OH}^{\cdot}
\end{aligned}
$$

representando a possível formação dos radicais hidroxila, que são as espécies responsáveis pela reação de caráter oxidativo do processo em estudo.

Baseado nos resultados obtidos na análise estatística foi realizado diferentes experimentos de FF utilizando as condiçôes ótimas de processo, ou seja: $\mathrm{pH}$ inicial em 2,3 e as concentraçôes $\mathrm{de}^{2 \mathrm{Fe}^{2+}=}$ $9,3 \mathrm{mg} \mathrm{L}^{-1}$ e $\mathrm{H}_{2} \mathrm{O}_{2}=65,5 \mathrm{mg} \mathrm{L}^{-1}$. (a)

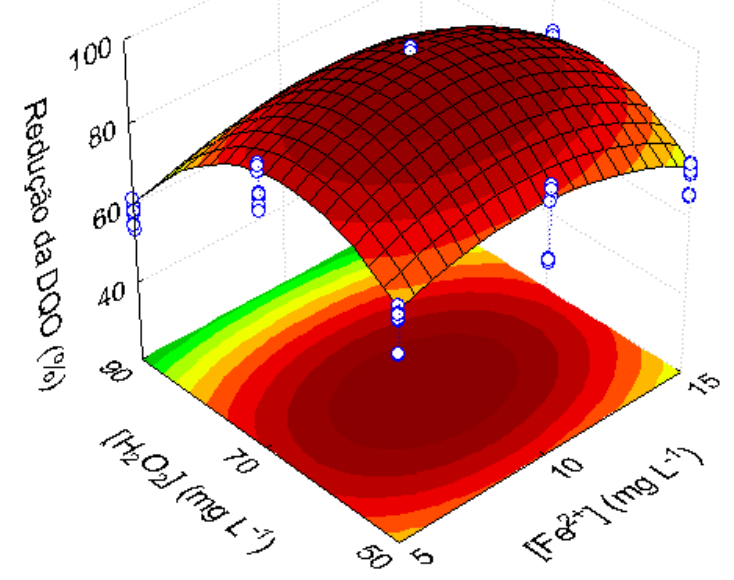

(b)

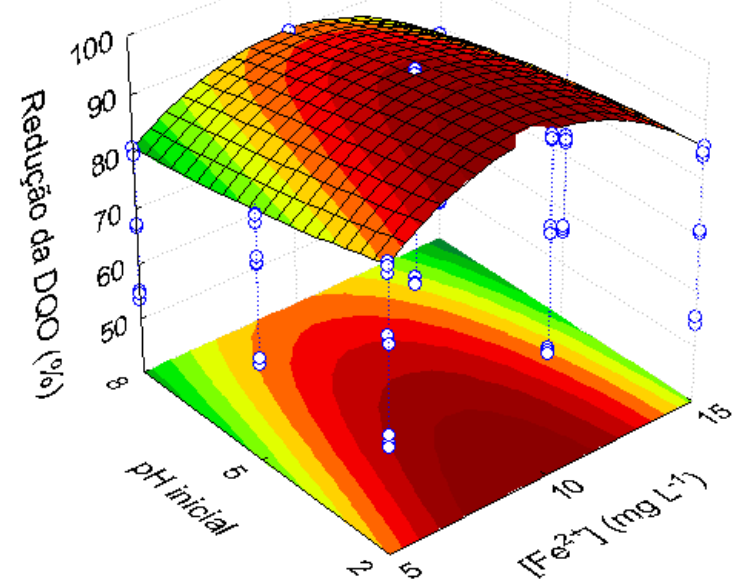

Figura 2 - Superfícies de resposta dos resultados experimentais obtidos no planejamento factorial $3^{3}$ completo para o processo FF, utilizando o ETS tratado, com os dados experimentais (a) redução da DQO em função da concentração de $\mathrm{Fe}^{2+}\left(\mathrm{q}_{1}\right)$ e concentração de $\mathrm{H}_{2} \mathrm{O}_{2}\left(\mathbf{q}_{2}\right)$ e $(\mathbf{b})$ redução da DQO em função da concentração de $\mathrm{Fe}^{2+}\left(\mathrm{q}_{1}\right)$ e $\mathrm{pH}$ inicial $\left(\mathrm{q}_{3}\right)$

Com o reator operando nestas condições foram retiradas alíquotas entre 5 e 90 minutos para verificar o efeito do tempo de irradiação para os melhores resultados na eficiência de redução da DQO, da turbidez, ST, STF e STV conforme apresentado na Figura 4. Após 5 minutos de tratamento via processo FF foi obtido o percentual de redução de 45\%, 8\%, 64\%, 91\% e 95\% dos valores de ST, STF, STV, DQO e turbidez, respectivamente.

Após 30 minutos os valores de redução permanecem praticamente constantes, obtendo cerca de $52 \%, 15 \%, 70 \%, 99 \%$ e $99,5 \%$ de remoção dos valores de ST, STF, STV, DQO e turbidez, respectivamente.

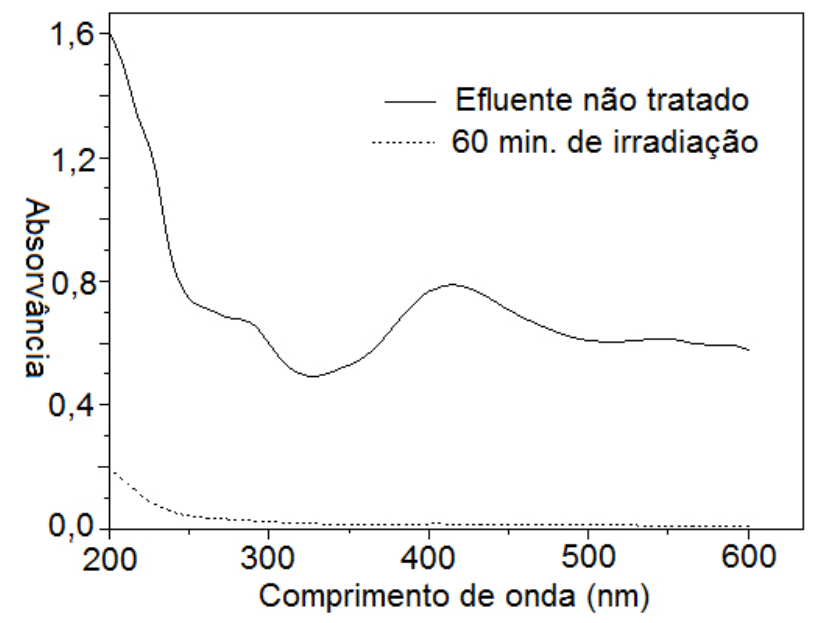

Figura 3 - Redução no valor da absorvância do ETS em função do tempo de irradiação 


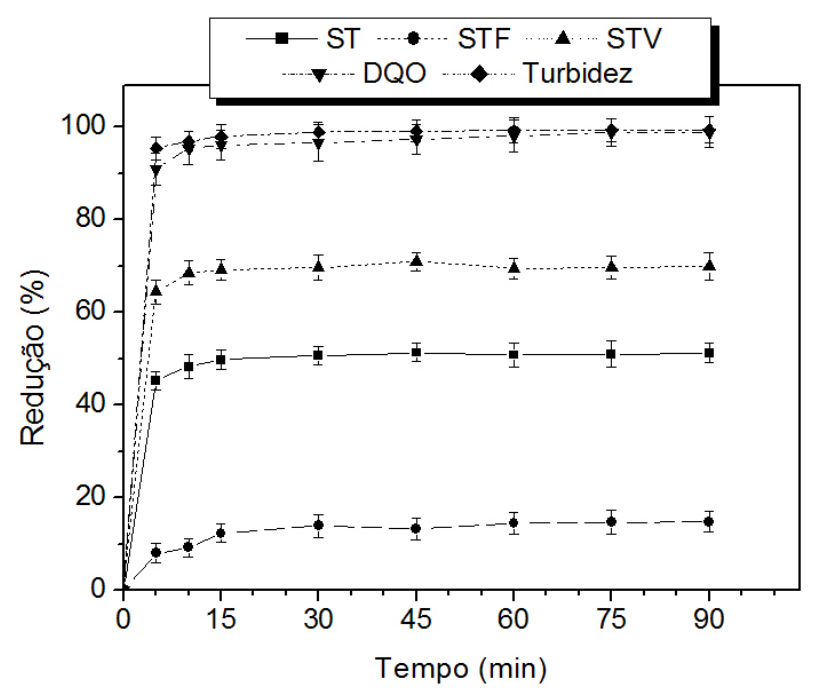

Figura 4 - Perfis de redução (ST, STF, STV, DQO e turbidez, \%) em função do tempo de irradiação no processo FF quando utilizado o ETS não-tratado com $\mathrm{pH}$ inicial em 2,3 e as concentrações de $\mathrm{Fe}^{2+}=9,3 \mathrm{mg} \mathrm{L}^{-1}$ e $\mathrm{H}_{2} \mathrm{O}_{2}=$ $65,5 \mathrm{mg} \mathrm{L}^{-1}$

A degradação dos corantes foi obtida com descoloração de 93\%, 93\%, 95\%, 96\% e 98\% para os corantes Amarelo, Laranja, Preto, Escarlate, Azul e Marinho, respectivamente. O percentual de descoloração nos comprimentos de onda de máxima absorção de cada corante em função do tempo de reação é apresentado na Figura 5.

Nos comprimentos de onda 228, 254, 284 e 310 é revelada a degradação dos compostos aromáticos em função dos efeitos do peróxido. A Figura 6 apresenta o percentual de degradação nos comprimentos de onda de máxima absorção dos compostos aromáticos em função do tempo de irradiação. A eficiência na degradação apresenta aumentos regulares em todos os comprimentos de onda, em proporçóes menores para o $228 \mathrm{~nm}$, por causa da interferência causada pelo $\mathrm{H}_{2} \mathrm{O}_{2}$ adicionado no início da reação. Após 60 minutos a eficiência de redução permanece constante obtendo $93 \%, 94 \%$, 96\% e $96,5 \%$ de redução da absorvância nos comprimentos de onda 228, 254, 284 e $310 \mathrm{~nm}$, respectivamente.

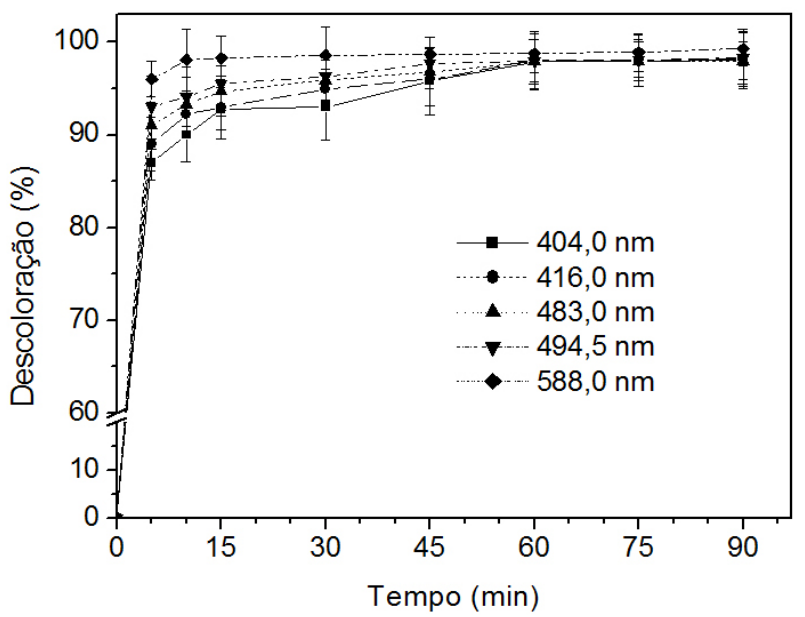

Figura 5 - Perfis de descoloração (\%) nos comprimentos de onda de máxima absorção de cada corante em função do tempo de irradiação no processo $\mathrm{FF}$, quando utilizado o ETS não-tratado com $\mathrm{pH}$ inicial em $2,3 \mathrm{e}$ as concentrações de $\mathrm{Fe}^{2+}=9,3 \mathrm{mg} \mathrm{L}^{-1}$ e $\mathrm{H}_{2} \mathrm{O}_{2}=$ $65,5 \mathrm{mg} \mathrm{L}^{-1}$

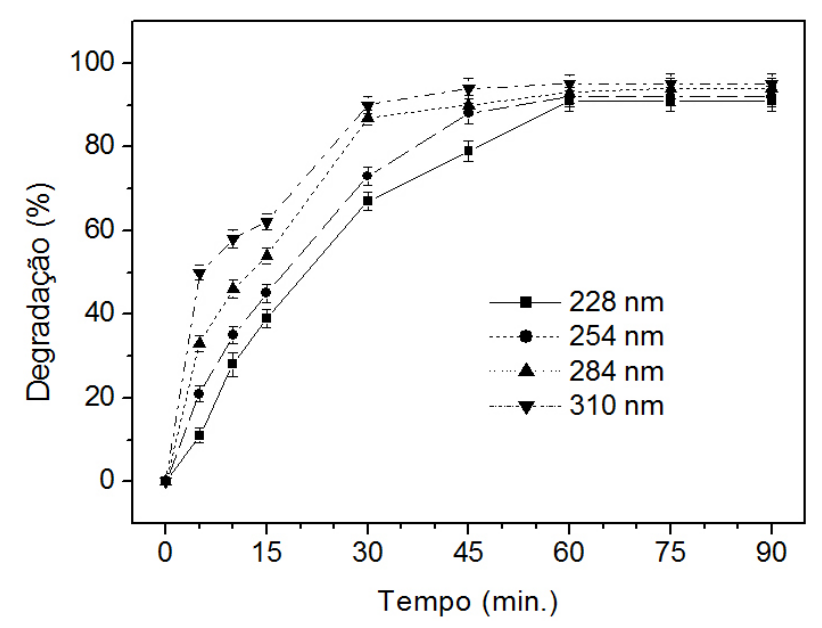

Figura 6 - Perfis de degradação (\%) nos comprimentos de onda de máxima absorção dos compostos aromáticos em função do tempo de irradiação, quando utilizado o ETS não-tratado com $\mathrm{pH}$ inicial em 2,3 e as concentrações de $\mathrm{Fe}^{2+}=9,3 \mathrm{mg} \mathrm{L}^{-1}$ e $\mathrm{H}_{2} \mathrm{O}_{2}=$

$$
65,5 \mathrm{mg} \mathrm{L}^{-1}
$$

Em geral, cinéticas de reações pseudo-primeira ordem podem predizer a evolução na degradação de substratos com propriedades persistentes (Konstantinou \& Albanis, 2004). A Tabela 9 apresenta o decaimento na absorbância para o ETS tratado via processo FF, em função das constantes da taxa de reação pseudo-primeira ordem e suas respectivas regressôes lineares nos comprimentos de onda dos compostos aromáticos. 
Tabela 9 - Porcentagem de degradação em 60 minutos de irradiação FF, constantes de reação

(k) e regressão linear (R) para o ETS.

\begin{tabular}{lcccc}
\hline ETS & $\begin{array}{c}228 \\
\mathrm{~nm}\end{array}$ & $\begin{array}{c}254 \\
\mathrm{~nm}\end{array}$ & $\begin{array}{c}284 \\
\mathrm{~nm}\end{array}$ & $\begin{array}{c}310 \\
\mathrm{~nm}\end{array}$ \\
\hline $\begin{array}{l}\text { Degradação } \\
(\%)\end{array}$ & 93 & 94 & 96 & 96,5 \\
$k\left(\mathrm{~min}^{-1}\right)$ & 0,048 & 0,064 & 0,073 & 0,090 \\
$\mathrm{R}$ & 0,997 & 0,991 & 0,993 & 0,992 \\
\hline
\end{tabular}

Esta mesma análise não foi realizada nos comprimentos de onda dos corantes devido a degradação ser muito rápida. A Figura 7 apresenta a regressão linear encontrada no estudo cinético proposto. Assumindo que para esta reação, o radical hidroxila e o efluente são determinantes para a taxa de reação, podemos utilizar a Equação (9):

$v=k_{\mathrm{OH}}\left[\mathrm{OH}^{*}\right] \mathrm{C}=k_{\text {ap }} \mathrm{C}$

onde C é a absorvância do efluente (Abs), $k_{\mathrm{OH}}$ é a constante da taxa de reação, e $k_{\text {ap }}$ é a constante de pseudo-primeira ordem. Assim, a linearização encontrada com ln (Abs x $100 / \mathrm{Abs}_{0}$ ) é confirmada (Malato, et al., 2003).

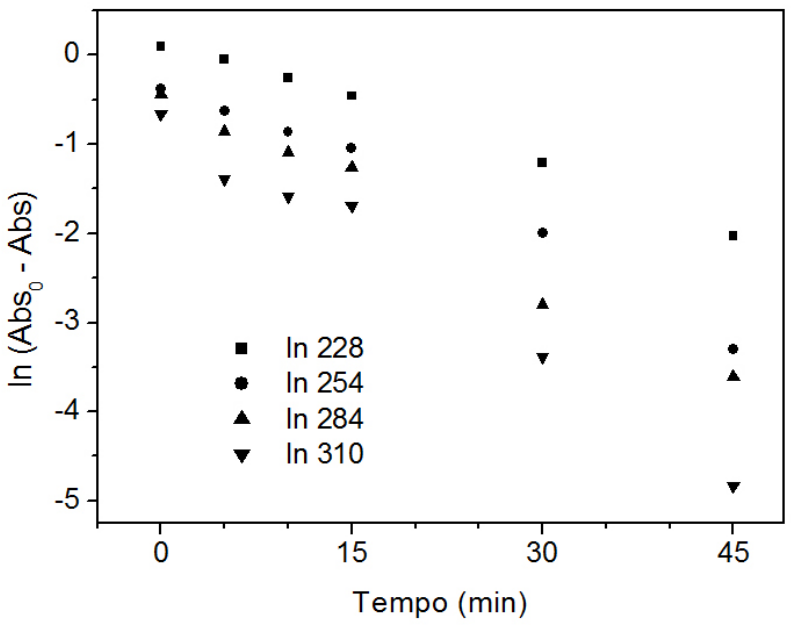

Figura 7 - Porcentagem de degradação do ETS em função do tempo de reação no processo FF para os comprimentos de onda de máxima absorção referentes aos compostos aromáticos

\section{CONCLUSÃO}

Baseado nos resultados obtidos no planejamento fatorial $3^{3}$ completo, as variáveis $\mathrm{pH}$ ini- cial e as concentrações de $\mathrm{Fe}^{2+}$ e $\mathrm{H}_{2} \mathrm{O}_{2}$ apresentam parâmetros estatisticamente significativos, há um nível de significância de 95\%, para a eficiência de degradação pelo processo FF do ETS. Foram obtidos resultados satisfatórios com o processo FF operando nas melhores condiçóes, sendo: $\mathrm{pH}$ inicial 2,3 e as concentraçôes de $\mathrm{Fe}^{2+}=9,3 \mathrm{mg} \mathrm{L}^{-1} \mathrm{e}$ $\mathrm{H}_{2} \mathrm{O}_{2}=65,5 \mathrm{mg} \mathrm{L}^{-1}$. Nestas condiçóes, os parâmetros físico-químicos e os corantes foram oxidados com apenas 5 minutos de irradiação, diferentemente dos compostos mais persistentes como os compostos aromáticos, que apresentaram resultados significantes de degradação com 60 minutos. Com o modelo estatístico e a cinética de reação propostos, o processo em estudo pode ser relacionado com a aplicação deste em escala real, já que os mesmos geraram um bom ajuste linear. Com os resultados apresentados, o processo FF pode ser considerado eficiente para a degradação dos compostos orgânicos podendo ser incorporado como parte dos sistemas de tratamento de efluentes tipicamente biológicos, já que estes caracterizamse essencialmente por uma simples transferência de fases dos poluentes, geralmente ocasionando acúmulos de resíduos sólidos como o lodo, que por sua vez, continua sendo um problema de poluição ambiental. Desta forma, o processo FF é alternativa eficaz para a degradação completa dos poluentes amenizando os impactos nos recursos hídricos bem como reduzindo contaminações por subprodutos finais do processo.

\section{AGRADECIMENTOS}

À CAPES e a Fundação Parque Tecnológico Itaipu - PTI C\&T/FPTI, pelo apoio financeiro.

\section{REFERÊNCIAS}

Cisneros, R.L., Espinoza, A.G., Litter, M.I., 2002. Photodegradation of an azo dye oh the textile industry. Chemosphere, 48, pp.393-399.

Habibi, M.H., Hassanzadeh, A., Mahdavi S., 2005. The effect of operational parameters on the photocatalytic degradation of three textile azo dyes in aqueous $\mathrm{TiO}_{2}$ suspensions. Journal Photochemistry Photobiological. A: Chem. 172, pp.89-96. 
Lucas, M.S., Peres, J.A., 2006. Decolorization of the azo dye Reactive Black 5 by Fenton and photo-Fenton oxidation. Dyes and Pigments. 71, pp.236-244.

Garcia, J.C., Simionato, J.I., da Silva, A.E.C., Nozaki, J., de Souza, N.E., 2009. Solar photocatalytic degradation of real textile effluents by associated titanium dioxide and hydrogen peroxide. Solar Energy. 83, pp.316-322.

Rivas, F.J., Carbajo, M., Beltrán, F., Gimeno, O., Frades, J., 2008. Comparison of different advanced oxidation processes (AOPs) in the presence of perovskites. Journal of Hazardous Materials. 155, pp.407-414.

Garcia, J.C., Oliveira, J.L., Silva, A.E.C., Oliveira, C.C., Nozaki, J., de Souza, N.E., 2007. Comparative study of the degradation of real textile effluents by photocatalytic reaction involving UV/ $\mathrm{TiO}_{2} / \mathrm{H}_{2} \mathrm{O}_{2}$ and $\mathrm{UV} / \mathrm{Fe}^{2+} / \mathrm{H}_{2} \mathrm{O}_{2}$ systems. Journal of Hazardous Materials. 147, pp.105-110.

Quici, N., Morgada, M.E., Gettar, R.T., Bolte, M., Litter, M.I., 2007. Photocatalytic degradation of citric acid under different conditions: $\mathrm{TiO}_{2}$ heterogeneous photocatalysis against homogeneous photolytic processes promoted by $\mathrm{Fe}(\mathrm{III})$ and $\mathrm{H}_{2} \mathrm{O}_{2}$. Applied Catalysis B: Environmental. 71, pp.117-124.

Liu, R., Chiu, H.M., Shiau, C., Yeh, R.Y., Hung, Y., 2007. Degradation and sludge production of textile dyes by Fenton and photo-Fenton processes. Dyes and Pigments. 73, pp.1-6.

Durán, A., Monteagudo, J.M., Amores, E., 2008. Solar photo-Fenton degradation of Reactive Blue
4 in a CPC reactor. Applied Catalysis B: Environmental. 80, pp.42-50.

APHA, AWWA, WPCF, 2005. Standard Methods for the Examination of Water and Wastewater, 21st ed., American Public Health Association, Washington, DC.

Kajitvichyanukul, P., Jamroensan, A., 2008. Formaldehyde degradation in the presence of methanol by photo-Fenton process. Journal of Environmental Management. 86, pp.545-553.

Knittel, D., Schollmeyer, E., 2008. Functional group analysis on oxidized surfaces of synthetic textile polymers. Journal of Hazardous Materials. 154, pp.83-91.

Makhotkina, O. S., Preis, S. V., Parkhomchuk, E. V., 2008. Water delignification by advanced oxidation processes: Homogeneous and heterogeneous Fenton and $\mathrm{H}_{2} \mathrm{O}_{2}$ photo-assisted reactions. Applied Catalysis B: Environmental. 74, pp.134140.

Primo, O., Rivero, M. J., Ortiz, I., 2008. PhotoFenton process as an efficient alternative to the treatment of landfill leachates. Journal of Hazardous Materials. 153, pp.834-842.

Konstantinou, I.K., Albanis, T.A., 2004. $\mathrm{TiO}_{2}-$ assisted photocatalytic degradation of azo dyes in aqueous solution: kinetic and mechanistic investigations: a review. Applied Catalysis B: Environmental. 49 pp.1-14.

Malato, S., Blanco, J., Vidal, A., Alarcón, D., Maldonado, M.I., Caceres, J., Gernjak, W., 2003. Applied studies in solar photocatalytic detoxification: an overview. Solar Energy. 75, pp.329-336. 